\title{
Relação do Tabagismo com Ansiedade e Depressão nos Cuidados de Saúde Primários
}

\author{
Relationship between Smoking and Anxiety and Depression in Primary Care
}

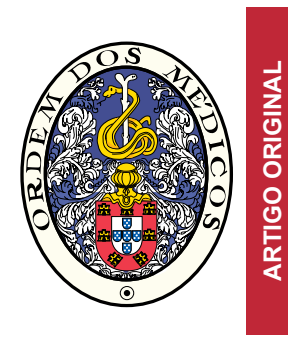

\author{
Helder FARINHA ${ }^{1}$, Joana RAPOSO DE ALMEIDA ${ }^{2}$, Ana Rita ALEIXO ${ }^{2,3}$, Hugo OLIVEIRA ${ }^{3,4}$, Filomena XAVIER ${ }^{5}$, Ana \\ Isabel SANTOS ${ }^{6}$ \\ Acta Med Port 2013 Sep-Oct;26(5):523-530
}

\section{RESUMO}

Introdução: A prevalência de tabagismo em Portugal é estimada em 19,7\% (2005). O tabagismo é prevalente nas perturbações da ansiedade. Alguns estudos referem que $60 \%$ dos fumadores tem história de depressão. A dependência tabágica pode ser avaliada pela escala de Fagerström. A Escala de Ansiedade e Depressão Hospitalar permite estimar a ansiedade e depressão. O objectivo deste trabalho foi procurar a existência de relação entre tabagismo e ansiedade/depressão em utentes de oito unidades de cuidados primários. Material e Métodos: Foi desenhado um estudo observacional, descritivo, transversal, analítico. Inquérito anónimo. Foram considerados como critérios de inclusão os utilizadores das unidades, maiores de 18 anos e alfabetizados e de exclusão os menores de 18 anos ou erros no preenchimento dos inquéritos. As variáveis consideradas foram: Fagerström, Escala de Ansiedade e Depressão Hospitalar, idade, estado civil, género, profissão, escolaridade.

Resultados: Foram considerados no estudo um total de 608 indivíduos, sendo $64 \%$ do género feminino e $21 \%$ de fumadores. Não verificámos diferenças em termos de ansiedade ou depressão na comparação entre não fumadores, ex-fumadores e fumadores. Verificámos que o grau de dependência da nicotina varia directamente com a ansiedade e depressão embora só se encontre relação estatisticamente significativa no género feminino, mesmo após correcção do efeito da idade.

Discussão: Existe relação entre dependência da nicotina e gravidade de sintomas de ansiedade e depressão, sobretudo no género feminino. Limitação: viés de selecção.

Conclusão: Este estudo fornece informação relativa aos factores psicológicos associados ao consumo de tabaco, podendo ser útil no tratamento da dependência de nicotina.

Palavras-chave: Ansiedade; Cuidados de Saúde Primários; Dependência do Uso de Tabaco; Depressão; Questionários; Tabagismo.

\section{ABSTRACT}

Introduction: Smoking prevalence in Portugal is estimated to be $19.7 \%$ (2005). Smoking is prevalent in anxiety disorders. Studies report that $60 \%$ of smokers have a history of depression. The Fagerström scale can be used to assess smoke dependence. The Hospital Anxiety and Depression Scale allows an estimate of anxiety and depression. Our goal was to find whether there is a relationship between smoking and anxiety / depression in eight clinics within primary care practice.

Material and Methods: We designed an observational, descriptive, cross-sectional, analytical study. Anonymous survey. We considered as inclusion criteria the over 18 years of age and literate clinic users and as exclusion criteria the under 18 years old users or incomplete surveys. We defined as variables: Fagerström, Hospital Anxiety and Depression Scale, age, marital status, gender, profession, schooling.

Results: We obtained a total of 608 valid surveys of which there were $64 \%$ women and $21 \%$ smokers. We found no differences in the prevalence of anxiety or depression when comparing non-smokers, ex-smokers and smokers. We found that the degree of nicotine addiction varies directly with anxiety and depression, however, the only statistically significant relationship observed was in women, even after correcting the effect of age.

Discussion: There is a relationship between nicotine dependence and the severity of symptoms of anxiety and depression, most relevant in women. Limitation: selection bias.

Conclusion: This study supplies information regarding psychological factors associated with tobacco consumption, allowing for its inclusion in treatment options for nicotine dependence.

Keywords: Anxiety Disorders; Depressive Disorder; Primary Health Care; Questionnaires; Smoking; Tobacco Use Disorder.

\section{INTRODUÇÃO}

As doenças mentais na Europa constituem uma causa importante de morbilidade, estando estimada uma prevalência de $27 \%$, dos quais $12 \%$ e $7,8 \%$ correspondem respectivamente às perturbações de ansiedade e do humor. ${ }^{1}$

Não existem dados disponíveis de base populacional que permitam estabelecer com fidedignidade a prevalência

dos vários tipos de perturbação depressiva ${ }^{2}$ ou de ansiedade em Portugal. Dois estudos antigos foram realizados na zona Centro, revelando que $16,9 \%$ a $18,4 \%$ da população apresentava sintomas depressivos significativos. ${ }^{3,4}$ Caldas de Almeida et al encontram-se na fase de preparação do Primeiro Estudo Nacional de Saúde Mental, recentemente

1. Unidade de Cuidados de Saúde Personalizados Covilhã Norte. Agrupamento de Centros de Saúde Cova da Beira. Covilhã. Portugal.

2. Unidade de Cuidados de Saúde Personalizados Fundão. Agrupamento de Centros de Saúde Cova da Beira. Fundão. Portugal.

3. Departamento de Medicina Geral e Familiar. Faculdade de Ciências da Saúde da Universidade da Beira Interior. Covilhã. Portugal.

4. Unidade de Cuidados de Saúde Personalizados 3 "Cuidar". Centro de Saúde de São Miguel. Unidade Local de Saúde de Castelo Branco. Castelo Branco. Portugal.

5. Unidade de Cuidados de Saúde Personalizados 1. Centro de Saúde de São Tiago. Unidade Local de Saúde de Castelo Branco. Castelo Branco. Portugal.

6. Unidade de Saúde Familiar "A Ribeirinha". Unidade Local de Saúde da Guarda. Guarda. Portugal.

Recebido: 20 de Novembro de 2012 - Aceite: 06 de Maio de 2013 | Copyright @ Ordem dos Médicos 2013 

ve. ${ }^{5}$

realizado no âmbito do 'World Mental Health Survey Initiati-

O consumo do tabaco atinge também valores importantes, com a prevalência do tabagismo a ser estimada em $19,7 \%$ (10\% são fumadores ocasionais e $89,3 \%$ fumadores diários) ${ }^{6}$

Há poucos estudos realizados em Portugal e nenhum com amostras com elevado número de elementos ou que explorem de forma sistemática a relação entre a ansiedade/depressão e hábitos tabágicos. Em 2008, Fernandes $M$ realizou um estudo comparativo entre fumadores e não fumadores, não tendo encontrado diferenças entre ansiedade e depressão nos dois grupos, embora a dependência, avaliada pelo teste de Fagerström, se relacionasse com os níveis de ansiedade e stresse. Este estudo, no entanto, apresentou uma amostra pequena e de conveniência, não representativa da população e com intervenção directa do investigador, não tendo sido feita uma apreciação crítica das limitações metodológicas. ${ }^{7}$ Dados publicados da população de uma consulta de cessação tabágica revelaram uma prevalência de patologia depressiva de $21,5 \%$ e de perturbação de ansiedade de $19,4 \%$, com $52 \%$ dos fumadores com tentativas de cessação infrutíferas a evocar a ansiedade como motivo de recaída. ${ }^{8}$

Segundo dados de alguns estudos internacionais, observa-se uma relação entre a ansiedade/depressão e hábitos tabágicos. No que diz respeito à relação entre a perturbação da ansiedade e o consumo de tabaco, mesmo tendo em consideração que a sua prevalência varia com o tipo de patologia da ansiedade em estudo e com a selecção da amostra, a perturbação de pânico, com ou sem agorafobia, tem sido associada a grandes prevalências de tabagismo (19-47\%), com as maiores prevalências desta associação a serem observadas no distúrbio de stresse pós-traumático (44-66\%). ${ }^{9}$

Por outro lado, o tabagismo é altamente prevalente em diversas perturbações da ansiedade. O consumo de tabaco aumenta o risco de desenvolvimento de ansiedade, com os fumadores a apresentam maiores taxas de perturbações da ansiedade que os não fumadores, com Odds Ratio (OR) entre 1,5 e 4,2. No entanto, é importante considerar a co-ocorrência de tabagismo com o consumo de álcool e outras substâncias, o que pode artificialmente inflacionar as estimativas de prevalência de tabagismo em indivíduos com perturbações de ansiedade.9,10

Os adolescentes são um grupo particularmente vulnerável ao consumo de tabaco. Por um lado, aqueles que fumam pelo menos um maço por dia têm um risco acrescido de desenvolver agorafobia $(\mathrm{OR}=6,8)$, distúrbio de pânico $(\mathrm{OR}=15,6)$ ou ansiedade generalizada $(\mathrm{OR}=5,5)$, mas não perturbação obsessivo-compulsiva ou ansiedade social no adulto jovem. ${ }^{11}$

Por outro lado, um estudo de Patton et al realizado em adolescentes, revelou que os que têm elevados níveis de ansiedade e depressão apresentam uma probabilidade duas vezes superior de serem fumadores, após terem sido eliminadas as variáveis de confundimento. A mesma rela- ção foi observada entre fumadores ocasionais e regulares, sendo que estes últimos apresentam uma probabilidade duas vezes superior de possuírem níveis de ansiedade e depressão elevados. ${ }^{12}$

Collins et al revelaram, num estudo realizado em populações de homens de meia-idade afro-americanos, que os fumadores apresentam maiores níveis de ansiedade que os antigos fumadores e os não fumadores, de forma estatisticamente significativa e que, de entre os fumadores, a maior carga tabágica se associa a maiores níveis de ansiedade, também de forma significativa. ${ }^{13}$

Relativamente aos mecanismos propostos para estas associações, observa-se que, se por um lado, a presença de ansiedade pode alterar a farmacocinética da nicotina, por outro, a precocidade do uso desta pode condicionar alterações neuroestruturais que podem modificar os efeitos da nicotina e/ou contribuir para manifestações de ansiedade..$^{9,14}$

No que diz respeito a depressão e tabagismo, a sua associação é conhecida. ${ }^{15}$ Cerca de $60 \%$ dos fumadores (homens e mulheres) têm história de depressão clínica. ${ }^{14}$ Um estudo de Epstein et al, que envolveu consumidores diários de tabaco e grandes consumidores de álcool mostrou, que qualquer um destes grupos apresenta um risco três vezes maior de apresentar depressão que os não consumidores de álcool e tabaco. ${ }^{16}$ Segundo um estudo de Kang et al, envolvendo mais de 27000 pessoas, o tabagismo encontra-se associado ao aparecimento de depressão, embora o inverso não tenha sido demonstrado. ${ }^{17}$

No entanto, segundo outros artigos publicados, a depressão parece estar fortemente associada a níveis de consumo tabágico elevados, embora não pareçam esclarecer se as pessoas que sofrem de depressão têm maior probabilidade de se tornarem fumadoras, pela procura dos efeitos da nicotina sobre o humor, ou se os sintomas depressivos são induzidos pelo consumo prolongado de tabaco, devido a alterações neuroquímicas. ${ }^{14}$

Embora se observe, de facto, uma relação entre ansiedade, depressão e tabagismo em vários estudos internacionais, não foi ainda possível identificar os factores comuns entre eles, assim como não se identificou o mecanismo de causa/efeito (se são a ansiedade e depressão que conduzem ao tabagismo ou se é o inverso). ${ }^{18}$

Tendo em consideração que não foram identificados estudos publicados em Portugal que explorem, de forma sistemática, a relação entre ansiedade e depressão com hábitos tabágicos, pretendemos avaliar a existência desta relação na população de utentes utilizadores de algumas unidades de cuidados de saúde primários (CSP) da Beira Interior.

São objectivos secundários determinar a prevalência e caracterizar quanto ao grau de dependência de tabagismo, a população de utentes utilizadores das unidades de CSP e, ainda, determinar a prevalência de sintomas ansiosos e depressão dessa mesma população. 


\section{MATERIAL E MÉTODOS}

Trata-se de um estudo observacional descritivo, transversal e analítico que incidiu, nas datas de aplicação dos inquéritos, sobre a população da área de influência de oito unidades de saúde da região da Beira Interior, distribuídas por quatro concelhos:

a) Castelo Branco: Unidade de Cuidados de Saúde Personalizados (UCSP) 1 e UCSP 2, do Centro de Saúde São Tiago, e UCSP 3, do Centro de Saúde São Miguel;

b) Covilhã: UCSP Covilhã 1 e UCSP Covilhã 2;

c) Fundão: UCSP Fundão Sede e UCSP Fundão extensões;

d) Guarda: Unidade de Saúde Familiar "A Ribeirinha".

Para tal, foi entregue um questionário a todos os utentes das referidas unidades de saúde, durante o período de 1 de Junho a 31 de Agosto de 2011, pelos funcionários administrativos das unidades de saúde.

O questionário incluía a avaliação do nível de ansiedade e depressão, usando a Escala de Ansiedade e Depressão Hospitalar (HADS) e a avaliação da dependência do tabaco, usando o teste de Fagerström (ambas validadas para a população portuguesa), ${ }^{19,20}$ para além de dados como a idade, estado civil, género, actividade profissional, escolaridade e uma questão versando o consumo de tabaco. Nesta questão ("Alguma vez fumou?") foram colocadas as três hipóteses de resposta "Sim, e fumo actualmente", "Sim, mas já deixei de fumar" e "Nunca", correspondendo respectivamente, para estudo analítico, a fumador, ex-fumador e não fumador.

A HADS ${ }^{19}$ é uma escala cuja versão em língua original se encontra sob direitos de autor e que se encontra validada para a população portuguesa, tendo sido autorizado o seu uso na versão portuguesa, de forma gratuita, pelo autor da validação em 25 de Outubro de 2010. Demora dois a cinco minutos a completar (auto-administração) e apesar de se denominar 'Hospitalar', a escala demonstrou ser válida em cuidados primários. ${ }^{19}$ Deve ser utilizada para rastreio, não para diagnóstico, e avalia dois itens: ansiedade e depressão, com pontuações distintas para cada perturbação (0-21 pontos cada escala). Na descrição do manual HADS a pontuação obtida pode ser categorizada em: sem patologia (0-7 pontos), ligeiro (8-10 pontos), moderado (1114 pontos), severo (11-14 pontos).

O teste de Fagerström ${ }^{20}$ é uma escala de seis itens desenhada para determinar a dependência do tabaco. Este teste mostrou boa consistência interna e relação positiva com variáveis-chave (ex: cotinina salivar) e altos graus de reprodutibilidade teste-reteste. A pontuação (0-10 pontos) pode ser categorizada em: dependência baixa (0-3 pontos); dependência média (4-6); dependência alta (7-10). Está validada para a população portuguesa e publicada em Open Access.

O questionário estava sujeito a preenchimento voluntário. A entrega do questionário foi feita pelo administrativo da unidade de saúde a todos os utentes da consulta, com posterior entrega do questionário preenchido em envelope fe- chado, sem identificação. Foi garantido não haver contacto directo com os investigadores, os quais exercem actividade clínica nas mesmas unidades.

Submetemos o protocolo à Comissão de Ética do Centro Hospitalar Cova da Beira e aos coordenadores das unidades de saúde, tendo sido aprovado pelos mesmos.

O estudo teve como critérios de inclusão a idade igual ou superior a 18 anos, a alfabetização dos utentes (ou analfabetos se auxiliados a preencher o inquérito por alguém exterior ao grupo de investigadores). Excluímos os inquéritos que não cumpriam os critérios de inclusão, os inquéritos com erros no preenchimento que invalidassem simultaneamente os dois componentes da escala HADS, inquéritos com informação incorrecta sobre história tabágica e os inquéritos de indivíduos fumadores com preenchimento inválido do teste de Fagerström.

Realizámos o cálculo amostral para se estimar o número mínimo de inquéritos válidos para que a amostra fosse significativa com um nível de confiança de $95 \%$ e uma margem de erro de $5 \%$, recorrendo à fórmula $n=\left(\right.$ N.p.q. $\left.\mathrm{V}^{2}\right) /$ (p.q. $\left.V^{2}+(N-1) \cdot E^{2}\right)$, em que: $N$ (população), $p$ (prevalência), $q=1-p ; V$ (valor crítico para intervalo de confiança de $95 \%$ ) $=1,96 ; \mathrm{E}$ (margem de erro) $=0,05$.

$\mathrm{O}$ valor $\mathrm{N}$ que considerámos foi de 152804 habitantes (maiores de 18 anos), distribuído da seguinte forma: Castelo Branco, com 46477 habitantes; Covilhã, com 44953 habitantes; Fundão, com 25914 habitantes; Guarda, com 35460 habitantes (dados fornecidos pelo Instituto Nacional de Estatística, referentes aos Censos 2001). Calculámos um número mínimo de inquéritos válidos necessários para que amostra fosse significativa de 383 inquéritos.

Distribuímos questionários em número superior ao necessário para conseguir uma amostragem representativa da população (1 521 inquéritos).

Para avaliar a relação entre género e a pontuação na escala HADS utilizámos o teste $U$ de Mann-Whitney. Fizemos a avaliação da relação entre a pontuação na escala de depressão ou a pontuação na escala de ansiedade, e a situação face ao tabaco, com recurso ao teste de Kruskal-Wallis. Estabelecemos a correlação entre variáveis quantitativas (HADS, idade e pontuação na escala de Fagerström) com recurso ao coeficiente de correlação de Pearson. Considerámos o nível de significância $(p)$ de $0,05 .{ }^{21}$

Elaborámos o registo de dados com recurso ao software Microsoft ${ }^{\circledR}$ Office Excel 2010 e o processamento estatístico com o programa SPSS 17.0.

\section{RESULTADOS}

Recebemos 724 envelopes. Destes, apenas 608 continham inquéritos que reuniam os critérios mínimos de inclusão, pelo que a amostra se considerou representativa da população. A amostra obtida, para um grau de confiança de 95\% apresenta uma margem de erro de $4 \%$.

A Tabela 1 apresenta a distribuição da amostra pelos quatro concelhos estudados.

A mediana da idade da população estudada foi de 46 anos, com um mínimo de 18 anos e um máximo de 88 
Tabela 1 - Características demográficas e sócio-económicas da amostra. Número de não fumadores, ex-fumadores e fumadores.

\begin{tabular}{|c|c|c|c|c|c|}
\hline \multirow[b]{2}{*}{ População } & \multirow[b]{2}{*}{$n=608$} & \multirow[b]{2}{*}{$\%$} & \multicolumn{3}{|c|}{ Situação face ao tabaco } \\
\hline & & & Não fumadores (n) & Ex-fumadores (n) & Fumadores (n) \\
\hline \multicolumn{6}{|l|}{ Concelho } \\
\hline Castelo Branco & 203 & 33,4 & 107 & 51 & 45 \\
\hline Covilhã & 183 & 30,1 & 94 & 53 & 36 \\
\hline Fundão & 178 & 29,3 & 84 & 51 & 43 \\
\hline Guarda & 44 & 7,2 & 27 & 12 & 5 \\
\hline \multicolumn{6}{|l|}{ Género } \\
\hline Masculino & 206 & 33,9 & 68 & 84 & 54 \\
\hline Feminino & 360 & 59,2 & 220 & 69 & 71 \\
\hline Não respondeu & 42 & 6,9 & 24 & 14 & 4 \\
\hline \multicolumn{6}{|l|}{ Estado civil } \\
\hline Solteiro & 108 & 17,8 & 45 & 27 & 36 \\
\hline Casado & 399 & 65,6 & 212 & 117 & 70 \\
\hline Divorciado & 33 & 5,4 & 11 & 12 & 10 \\
\hline Viúvo & 27 & 4,4 & 22 & 5 & 0 \\
\hline União de facto & 25 & 4,1 & 11 & 3 & 11 \\
\hline Não respondeu & 16 & 2,6 & 11 & 3 & 2 \\
\hline \multicolumn{6}{|l|}{ Actividade profissional } \\
\hline Rural & 6 & 1,0 & 5 & 1 & 0 \\
\hline Comércio & 75 & 12,3 & 26 & 30 & 19 \\
\hline Indústria & 63 & 10,4 & 30 & 15 & 18 \\
\hline Serviços & 182 & 29,9 & 76 & 55 & 51 \\
\hline Doméstico(a) & 47 & 7,7 & 38 & 7 & 2 \\
\hline Estudante & 41 & 6,7 & 20 & 8 & 13 \\
\hline Reformado & 119 & 19,6 & 73 & 39 & 7 \\
\hline Desempregado & 52 & 8,6 & 27 & 8 & 17 \\
\hline Não respondeu & 23 & 3,8 & 17 & 4 & 2 \\
\hline \multicolumn{6}{|l|}{ Formação escolar } \\
\hline Não sabe ler ou escrever & 2 & 0,3 & 0 & 2 & 0 \\
\hline $\begin{array}{l}\text { Sabe ler ou escrever mas tem menos de } \\
4 \text { anos de escolaridade }\end{array}$ & 13 & 2,1 & 11 & 0 & 2 \\
\hline 4 anos de escolaridade & 90 & 14,8 & 57 & 26 & 7 \\
\hline Entre 5 e 9 anos de escolaridade & 180 & 29,6 & 92 & 48 & 40 \\
\hline Entre 10 e 12 anos de escolaridade & 166 & 27,3 & 73 & 53 & 40 \\
\hline Bacharelato / Licenciatura & 114 & 18,8 & 47 & 31 & 36 \\
\hline Mestrado / Doutoramento & 21 & 3,5 & 13 & 6 & 2 \\
\hline Não respondeu & 22 & 3,6 & 19 & 1 & 2 \\
\hline
\end{tabular}

anos. Na Tabela 1 também se encontram as características sócio-demográficas desta amostra. Verificámos um predomínio do género feminino, utentes casados, escolarizados e em situação activa de emprego. Alguns grupos são fracamente representados, o que impediu a comparação entre os diferentes grupos sócio-económicos.

Na Tabela 2 descrevemos os perfis de sintomas ansiosos e depressivos (estratificados pelas pontuações descritas segundo o manual HADS), ${ }^{19}$ a percentagem de não fumadores, ex-fumadores e fumadores, assim como os graus de dependência estratificados pelas pontuações da escala de Fagerström descritas na validação da escala portuguesa. ${ }^{12}$

Pesquisámos a existência de variações na escala HADS em relação com as variáveis género e idade.

Não encontrámos relação entre a pontuação na escala de ansiedade da HADS e a idade (coeficiente de determinação $\left(R^{2}\right)$ de 0,001 e $\left.p=0,454\right)$.

Encontrámos diferenças entre a pontuação na escala de ansiedade da HADS e o género. O valor médio de 
Tabela 2 - Estratificação da amostra na Escala de Ansiedade e Depressão Hospitalar e situação face ao tabaco. Estratificação do grau de dependência nos fumadores.

\begin{tabular}{|c|c|c|}
\hline Perfil de sintomas ansiosos (escala da HADS-ansiedade) & $n=608$ & $\%$ \\
\hline Sem patologia (0-7 pontos) & 243 & 40,0 \\
\hline Ansiedade ligeira (8-10 pontos) & 160 & 26,3 \\
\hline Ansiedade moderada (11-14 pontos) & 132 & 21,7 \\
\hline Ansiedade severa (15-21 pontos) & 54 & 8,9 \\
\hline Erro no preenchimento da escala & 19 & 3,1 \\
\hline Perfil de sintomas depressivos (escala da HADS-depressão) & $n=608$ & $\%$ \\
\hline Sem patologia (0-7 pontos) & 406 & 66,8 \\
\hline Depressão ligeira (8-10 pontos) & 114 & 18,8 \\
\hline Depressão moderada (11-14 pontos) & 42 & 6,9 \\
\hline Depressão severa (15-21 pontos) & 23 & 3,8 \\
\hline Erro no preenchimento da escala & 23 & 3,8 \\
\hline Situação face ao tabaco & $n=608$ & $\%$ \\
\hline Não fumador & 312 & 51,3 \\
\hline Ex-fumador & 167 & 27,5 \\
\hline Fumador & 129 & 21,2 \\
\hline Grau de dependência nos fumadores (escala de Fagerström) & $n=129$ & $\%$ \\
\hline Dependência baixa (0-3 pontos) & 75 & 58,1 \\
\hline Dependência média (4-6 pontos) & 43 & 33,3 \\
\hline Dependência alta (7-10 pontos) & 11 & 8,5 \\
\hline
\end{tabular}

Tabela 3 - Relação entre situação face ao tabaco e pontuação média na Escala de Ansiedade e Depressão Hospitalar. Comparação inter-grupos.

\begin{tabular}{|c|c|c|c|c|}
\hline & Situação face ao tabaco & $\mathbf{n}$ & Média da escala HADS (IC=95\%) & $p$ \\
\hline \multicolumn{5}{|c|}{ Situação face ao tabaco e ansiedade. Análise por géneros. } \\
\hline \multirow{3}{*}{ Género masculino e feminino } & Não fumador & 299 & $8,96(8,48-9,45)$ & \multirow{3}{*}{0,094} \\
\hline & Ex-fumador & 162 & $8,05(7,41-8,69)$ & \\
\hline & Fumador & 128 & $8,84(8,11-9,56)$ & \\
\hline \multirow{3}{*}{ Género masculino } & Não fumador & 65 & $7,34(6,53-8,14)$ & \multirow{3}{*}{0,595} \\
\hline & Ex-fumador & 81 & $7,11(6,22-8,00)$ & \\
\hline & Fumador & 53 & $7,43(6,55-8,32)$ & \\
\hline \multirow{3}{*}{ Género feminino } & Não fumador & 212 & $9,44(8,86-10,02)$ & \multirow{3}{*}{0,913} \\
\hline & Ex-fumador & 68 & $9,34(8,36-10,31)$ & \\
\hline & Fumador & 71 & $9,70(8,65-10,76)$ & \\
\hline \multicolumn{5}{|c|}{ Situação face ao tabaco e depressão. Análise por géneros. } \\
\hline \multirow{3}{*}{ Género masculino e feminino } & Não fumador & 299 & $6,13(5,67-6,59)$ & \multirow{3}{*}{0,053} \\
\hline & Ex-fumador & 161 & $5,37(4,76-5,99)$ & \\
\hline & Fumador & 125 & $5,19(4,55-5,84)$ & \\
\hline \multirow{3}{*}{ Género masculino } & Não fumador & 64 & $5,75(4,78-6,72)$ & \multirow{3}{*}{0,390} \\
\hline & Ex-fumador & 82 & $5,44(4,49-6,39)$ & \\
\hline & Fumador & 51 & $4,67(3,76-5,57)$ & \\
\hline \multirow{3}{*}{ Género feminino } & Não fumador & 213 & $6,22(5,65-6,78)$ & \multirow{3}{*}{0,158} \\
\hline & Ex-fumador & 65 & $5,02(4,14-5,89)$ & \\
\hline & Fumador & 70 & $5,47(4,52-6,42)$ & \\
\hline
\end{tabular}


pontuação para o género masculino foi de 7,27 $(n=199$; intervalo de confiança a $95 \%$ para a média (IC95) foi de $6,77-7,77)$ e para o género feminino foi de $9,47(n=351$; IC95 = 9,02-9,92). O valor de $p$ que obtivemos foi $<0,001$.

Encontrámos uma relação positiva entre a pontuação na escala de depressão da HADS e a idade, com $\mathrm{R}^{2}=$ 0,0981 e $p<0,001$.

Não encontrámos diferenças entre a pontuação na escala de depressão da HADS e o género. $O$ valor médio de pontuação para o género masculino foi de $5,34(n=197$; IC95 $=4,79-5,89)$ e para o género feminino foi de $5,84(n=$ 348 ; IC95 $=5,42-6,27$ ). O valor de $p$ que obtivemos foi de 0,144 .

$\mathrm{Na}$ Tabela 3 apresentamos as comparações para as pontuações nas escalas da HADS para não fumadores, ex-fumadores e fumadores, separadas por género. Não encontrámos diferenças estatisticamente significativas.

$\mathrm{Na}$ Fig. 1 mostramos a relação entre a dependência do tabaco nos fumadores, avaliada pela escala de Fagers- tröm, e a pontuação na escala de ansiedade da HADS. Avaliámos a variação por regressão estatística, procedendo à análise por género. A correlação estatística foi estatisticamente significativa para o género feminino $(p=0,028)$, com $p=0,160$ para o género masculino e $p=0,073$ para ambos os géneros. Na figura mostramos as equações da regressão linear, assim como o coeficiente de determinação.

Na Fig. 2 mostramos a relação entre a dependência de tabaco nos fumadores, avaliada pela escala de Fagerström, e a pontuação na escala de depressão da HADS. Também aqui avaliámos a variação por regressão linear e fizemos a análise por género. A correlação estatística foi significativa para o género feminino, mesmo após correcção do factor idade ( $p<0,001$ em ambos os casos) e para a globalidade da amostra ( $p=0,008$, o qual após correcção do factor idade é de $p=0,019$ ). Encontrámos um valor não significativo para o género masculino $(p=0,640)$.

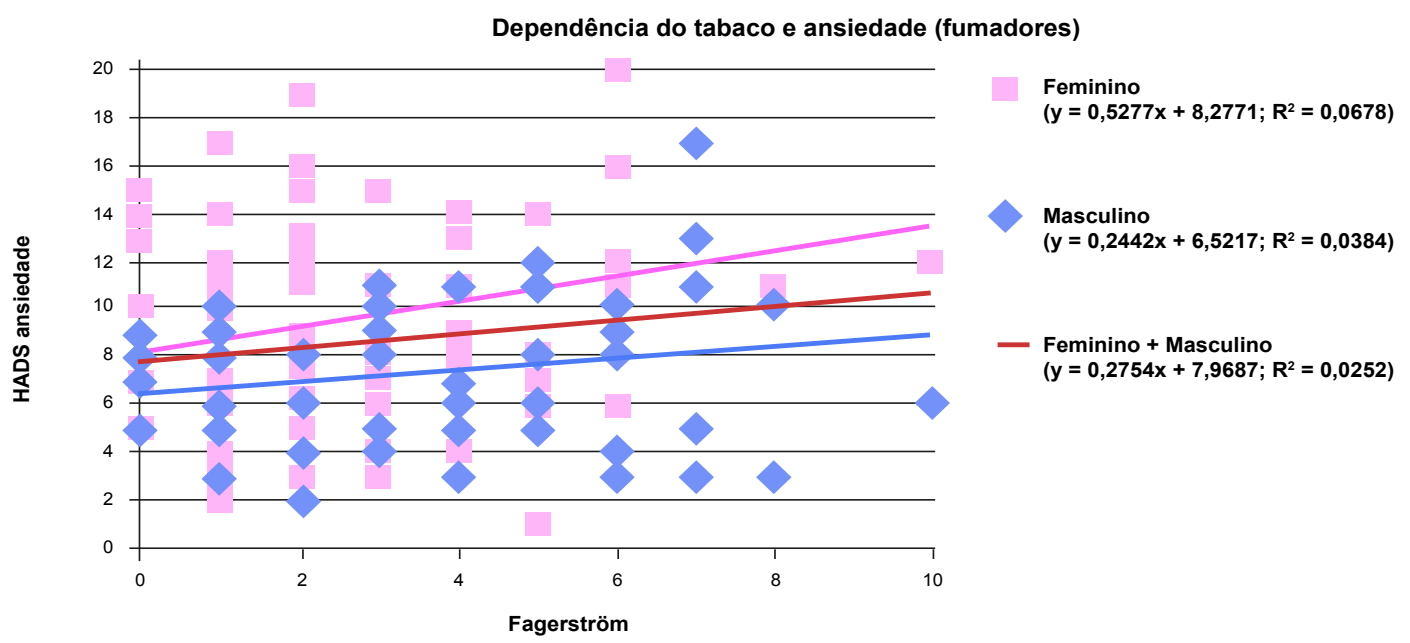

Figura 1 - Relação entre dependência do tabaco (Fagerström) e ansiedade. Variação avaliada por regressão linear entre ansiedade e dependência do tabaco. Análise por géneros. Relação estatística (Pearson) significativa apenas para o género feminino $(p=0,028) ; p$ para o género masculino e fumadores em geral de 0,160 e 0,073 .

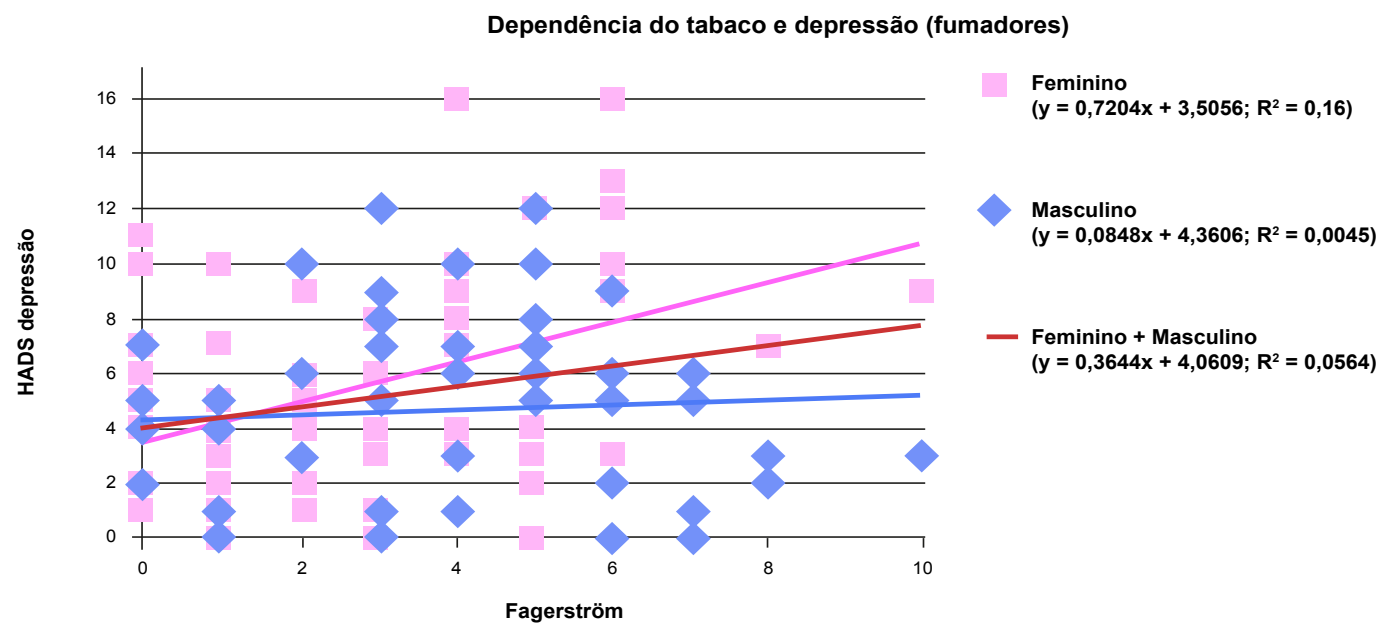

Figura 2 - Relação entre dependência do tabaco (Fagerström) e depressão. Variação avaliada por regressão linear entre depressão e dependência do tabaco. Análise por géneros. Relação estatística (Pearson) significativa para o género feminino, mesmo após correç̧ão do factor idade $(p=0,001)$ e para a globalidade da amostra $(p=0,008$; após correcção do factor idade $p=0,019)$. Valor para o género masculino $p=0,640$. 


\section{DISCUSSÃO}

Consideramos que a metodologia que utilizámos neste estudo foi a mais conveniente, apesar de poder apresentar como limitação um viés de selecção. A amostra que utilizámos neste estudo corresponde a utentes do Serviço Nacional de Saúde, nomeadamente das oito unidades de saúde enumeradas na metodologia, pelo que esta amostra pode ser pouco representativa da população-alvo deste estudo, relativa à área de influência dessas mesmas unidades de saúde. Este viés na escolha da amostra condiciona a fraca representatividade de alguns grupos populacionais e a aplicação destes resultados a outras regiões que não as estudadas. É de notar ainda o viés de voluntarismo neste trabalho de investigação, uma vez que concedemos aos utentes a liberdade de resposta ao questionário. A própria escala HADS, validada para a população portuguesa no trabalho publicado por Ribeiro et al em $2007,{ }^{19}$ não se trata de uma escala de diagnóstico de perturbações de humor ou de ansiedade, podendo apenas ser utilizada como um método de rastreio. Desta forma, os valores que encontrámos apenas podem ser interpretados como uma estimativa da gravidade dos sintomas relacionados com depressão ou ansiedade. Outra limitação do estudo passa pela incompleta definição do critério de ex-fumador, uma vez que não definido um limite temporal de abstinência tabágica a partir do qual se considera ex-fumador.

Obtivemos uma amostra significativa da população. A predominância do género feminino pode dever-se a uma possível maior taxa de utilização da consulta no Centro de Saúde por parte deste género, em comparação com o género masculino, tal como verificado noutros estudos realizados em cuidados primários. ${ }^{22-24}$ No que diz respeito à escolaridade da amostra estudada, $82 \%$ dos inquiridos apresentam habilitações superiores a cinco anos de frequência escolar. Tal situação pode-se ter apresentado pelo facto de termos estabelecido o analfabetismo como um critério de exclusão à participação neste estudo, ou pela dificuldade no preenchimento do questionário por indivíduos com habilitações inferiores.

A prevalência de tabagismo que observámos nesta amostra foi de $21 \%$, ligeiramente superior ao descrito no Inquérito Nacional de Saúde (dados de 2005/2006), ${ }^{6}$ onde se encontrava uma prevalência de $19,7 \%$ para a população nacional e de $16,1 \%$ para a população da Região Centro, tendo presente que podemos encontrar nestes resultados o viés de voluntarismo. De entre os fumadores, a predominância de utentes com baixo grau de dependência foi também verificada no estudo de John et al, ${ }^{25}$ embora neste estudo o número de fumadores que pontuaram entre 8 e 10 pontos no teste de Fagerström fosse muito inferior $(2,2 \%)$ comparativamente à nossa amostra $(8,5 \%)$. Estes valores podem ter por causa o número de fumadores incluídos nos estudos ou diferenças da estrutura das populações.

Os resultados que encontrámos neste estudo não mostram uma relação entre ser ou não fumador e sintomas de ansiedade ou depressão, o que vem ao encontro da grande maioria das publicações encontradas. Embora o estudo de
Son et al publicado em $1997^{15}$ tenha demostrado diferenças na prevalência dos sintomas de depressão entre fumadores e não fumadores, estudos mais recentes, incluindo um trabalho realizado por Fernandes $\mathrm{M}$ em $2008^{7}$ e aplicado a uma amostra de população portuguesa, vêm sugerir que, de uma forma geral, não existem diferenças estatisticamente significativas nos níveis de ansiedade e depressão entre os indivíduos fumadores e os indivíduos não fumadores, embora com as limitações já descritas anteriormente. O próprio estudo de Son et $\mathrm{al}^{15}$ estava circunscrito a uma população muito jovem, com características de raça e idade que impedem uma comparação directa. No entanto, podemos observar que os níveis de dependência, analisados através do questionário de Fagerström, e os níveis de ansiedade e depressão, segundo a escala de HADS, estão correlacionados significativamente, ou seja, quanto maior for a dependência tabágica, mais elevados serão os níveis de depressão, tal como descrito por Nunes E em 2006, ${ }^{14}$ bem como os níveis de ansiedade. Esta relação entre a dependência da nicotina e a gravidade dos sintomas de ansiedade e depressão também foi observada na amostra estudada, apresentando uma variação positiva para todos os grupos estudados, embora apenas tenhamos verificado uma relação estatisticamente significativa para o género feminino e mais pronunciada no que diz respeito aos sintomas de depressão.

Neste estudo, não foi possível estabelecermos uma relação de causa ou efeito entre o início dos hábitos tabágicos e a presença dos primeiros sintomas de ansiedade ou depressão. Esta relação foi evidente em alguns estudos, como o da autoria de Morissette et al publicado em 2007, ${ }^{9}$ que mostrou uma relação temporal entre o tabagismo e a perturbação de ansiedade, verificando-se uma elevada taxa de fumadores entre a população com perturbações da ansiedade, mas surgindo também a dependência de nicotina como um factor de risco para o desenvolvimento de perturbações de ansiedade, especialmente em adultos jovens. Nesse artigo também é referido que adolescentes com consumo de tabaco têm maior risco de desenvolver alguns tipos de perturbação de ansiedade, embora o inverso também se possa verificar. Tal como sugerido por outros autores, como por exemplo por Kang et al na sua publicação de $2010,{ }^{17}$ é necessária a realização de estudos longitudinais para analisar a relação de causalidade entre os hábitos tabágicos e a prevalência de sintomas de depressão e ansiedade.

Este estudo fornece informação que permite alargar o conhecimento sobre os factores psicológicos associados ao consumo de tabaco, sendo importante para a prática clinica em Medicina Geral e Familiar, essencialmente no tratamento do doente dependente de nicotina ou com perturbações de ansiedade e depressão.

\section{CONCLUSÃO}

Os resultados deste estudo não mostram uma relação entre ser fumador, ex-fumador ou não fumador com sintomas de ansiedade ou depressão. A investigação efectuada 
sugere, no entanto, que existe uma relação entre a dependência da nicotina e a gravidade dos sintomas de ansiedade e depressão, essencialmente para o género feminino. Na relação entre os hábitos tabágicos e ansiedade/depressão não nos foi possível identificar qual é causa e qual é o efeito.

Os resultados deste estudo devem ser confirmados, no entanto, através de estudos que consigam ultrapassar as limitações verificadas, nomeadamente desenhando um estudo com uma maior amostra e abrangência geográfica, com maior representação dos diferentes grupos populacionais e que permita obviar os vieses ou outras limitações identificados.

\section{REFERÊNCIAS}

1. Wittchen HU, Jacobi F. Size and burden of mental disorders in Europe - a critical review and appraisal of 27 studies. Eur Neuropsychopharmacol. 2005;15:357-76.

2. Gusmão RM, Xavier M, Heitor MJ, Bento A, Caldas de Almeida JM. O peso das perturbações depressivas: aspectos epidemiológicos globais e necessidades de informação em Portugal. Acta Med Port. 2005;18:129-46.

3. Serra V, Firmino AC. Estados de tensão emocional, solidão e sintomas depressivos na população em geral. Psiquiatr Clin. 1989;10:149-55

4. Serra V, Matos AP, Gonçalves S. Auto-conceito e sintomas depressivos na população em geral. Psiquiatr Clin. 1987;7:97-102.

5. Direção-Geral da Saúde. Programa Nacional Para a Saúde mental, Reatualização do Plano Nacional de Saúde Mental; [Consultado 2013 Abr 24]. Disponível em http://www.saudemental.pt/wp-content/uploads/2012/06/Recalendarizac\%CC\%A7a\%CC\%83o_PNSM.pdf.

6. Instituto Nacional de Saúde Dr. Ricardo Jorge (INSA) e Instituto Nacional de Estatística (INE). $4^{\circ}$ Inquérito Nacional de Saúde 2005-2006; [Consultado 2011 Out 11] Disponível em http://www.insa.pt/sites/INSA Portugues/Publicacoes/Outros/Paginas/INS2005-2006.aspx.

7. Fernandes MP. Ansiedade, depressão, stress e tabagismo - Estudo comparativo em indivíduos fumadores e indivíduos não fumadores. Trabalho para obtenção do grau de licenciatura em Psicologia. Universidade Fernando Pessoa, 2008. [Consultado 2012 Set 16] Disponível em http://bdigital.ufp.pt/bitstream/10284/1466/1/MONO_1102.pdf.

8. Aguiar M, Todo-Bom F, Felizardo M, Macedo R, Caeiro F, Sotto-Mayor $\mathrm{R}$, et al. Casuística de quatro anos de uma consulta de apoio ao fumador. Rev Port Pneumol. 2009;15:179-97.

9. Morissette SB, Tull MT, Gulliver SB, Kamholz BW, Zimering RT. Anxiety, anxiety disorders, tobacco use, and nicotine: A critical review of interrelationships. Psychol Bull. 2007;133:245-72.

10. Black DW, Zimmerman M, Coryell WH. Cigarette smoking and psychiatric disorder in a community sample. Ann Clin Psychiatry. 1999;11:129 36.

11. Johnson JG, Cohen P, Pine DS, Klein DF, Kasen S, Brook JS. Association between cigarette smoking and anxiety disorders during adolescence and early adulthood. JAMA. 2000;284:2348-51.

12. Patton GC, Hibbert M, Rosier MJ, Carlin JB, Caust J, Bowes G. Is smok-

\section{CONFLITO DE INTERESSES}

Os autores declaram que não houve conflito de interesses na realização deste trabalho.

\section{FONTES DE FINANCIAMENTO}

Os autores declaram a inexistência de bolsas. Os custos com a investigação foram suportados pelos próprios e usando os recursos próprios das unidades de saúde. Esta investigação foi apresentada, em parte, sob a forma de poster, no evento "Update em Medicina", que decorreu no Hotel Grande Real Santa Eulália - Albufeira, nos dias 3 a 6 de Maio de 2012.

ing associated with depression and anxiety in teenagers? Am J Public Health. 1996;86:225-30.

13. Collins BN, Lepore SJ. Association between anxiety and smoking in a sample of urban black men. J Immigr Minor Health. 2009;11:29-34.

14. Nunes E. Consumo de tabaco. Efeitos na saúde. Rev Port Clin Geral. 2006;22:225-44.

15. Son BK, Markovitz JH, Winders S, Smith D. Smoking, nicotine dependence, and depressive symptoms in the CARDIA Study. Am J Epidemiol. 1997;145:110-6.

16. Epstein JF, Induni M, Wilson T. Patterns of clinically significant symptoms of depression among heavy users of alcohol and cigarettes. Prev Chronic Dis. 2009;6:A09.

17. Kang $E$, Lee J. A longitudinal study on the causal association between smoking and depression. J Prev Med Public Health. 2010;43:193-204.

18. Morrell HE, Cohen LM. Cigarette smoking, anxiety, and depression. J Psychopathol Behav Assess. 2006;28:281-329.

19. Pais-Ribeiro J, Silva I, Ferreira T, Martins A, Meneses R, Baltar M. Va lidation study of a Portuguese version of the Hospital Anxiety and Depression Scale. Psychol Health Med. 2007;12:225-37.

20. Ferreira LF, Quintal C, Lopes I, Taveira N. Teste de dependência à nicotina: validação linguística e psicométrica do teste de Fagerström. Rev Port Saúde Pública. 2009;27:37-56.

21. Maroco J. Análise estatística - com utilização do SPSS. $3^{a}$ ed. Lisboa: Edições Sílabo; 2007.

22. Ferreira PL, Raposo V. A governação em saúde e a utilização de indicadores de satisfação. Rev Port Clin Geral. 2006;22:285-96.

23. Ferreira PL, Luz A, Valente S, Raposo V, Godinho P, Felício ED. Determinantes da satisfação dos utentes dos cuidados primários: o caso de Lisboa e Vale do Tejo. Rev Port Saúde Pública. 2001;2:53-61.

24. Hespanhol A, Ribeiro O, Costa-Pereira A. Assegurar qualidade no Centro de Saúde São João. Satisfação dos utentes. Arq Med. 2005;19:1917.

25. John U, Meyer C, Hapke U, Rumpf H-J, Schumann A, Adam C, et al The Fagerström test for nicotine dependence in two adult population samples - potential influence of lifetime amount of tobacco smoked on the degree of dependence. Drug Alcohol Depend. 2003;71:1-6. 